\title{
THE ELONGATION OF ABELL CLUSTERS. III. RICH CLUSTERS AND INITIAL CONDITIONS
}

\author{
GEORGE RHEE
}

New Mexico State University, Department of Astronomy, Box 30001/Department 4500, Las Cruces, New Mexico 88003

\author{
Michael van HaArlem and Peter Katgert
}

Leiden Observatory, Postbus 9513, 2300 RA Leiden, The Netherlands

Received 31 October 1991; revised 13 February 1992

\begin{abstract}
We present the results of a study of the elongation and orientation of a sample of 107 rich clusters of galaxies. The data consist of galaxy lists derived from digitized images of the Palomar Sky Survey red plate copies. We work with three measures of the cluster orientation (1) the cluster position angle derived from the Fourier transform of the azimuthal galaxy distribution, (2) the first-ranked galaxy position angle derived from the moments of the digitized galaxy image, and (3) the cluster position angle derived from the $\mathrm{x}$-ray surface brightness distribution [Einstein data]. We have examined the orientation of clusters with respect to their nearest neighbors as a function of distance to the nearest neighbor. The orientation of clusters with respect to members of the same supercluster has also been examined. We find that alignment effects are present for clusters residing in superclusters. We also find that $\mathrm{cD}$ galaxies are significantly aligned with the direction to the nearest neighbor cluster. These results are discussed in the context of current ideas on galaxy formation.
\end{abstract}

\section{INTRODUCTION}

In this paper, we present results of a study of the elongation and orientation of a sample of 107 rich clusters of galaxies. The goal of this work is to use the high density peaks in the galaxy distribution to differentiate between various fluctuation spectra and to constrain the physics of the universe prior to galaxy formation. In this section of the paper, we review the theoretical motivation for this work and the previous observational work which has been done on alignments. A value of $H_{0}$ of $50 \mathrm{~km} / \mathrm{s} / \mathrm{Mpc}$ is used in this paper.

\subsection{Theoretical Background}

On the theoretical front, a number of properties of galaxy clusters have been studied in a set of papers reporting results of $N$-body simulations by West and collaborators (1987, 1988a,b, 1989). The goal of this research program is to study the growth of small fluctuations due to purely gravitational clustering in an expanding universe. These initial fluctuations are assumed to have grown to produce the large scale structure seen today. Depending on the exact form of the initial fluctuation spectrum, structure may have formed either by clustering hierarchically from small to large scales, or via fragmentation from large to small scales. The initial form of the fluctuation spectrum is determined by the nature of the nondissipative dark matter. For hot dark matter, free streaming destroys all but the largest fluctuations of supercluster size. For cold dark matter, free streaming is cosmologically irrelevant and structures form by clustering hierarchically from small to large scales (Peebles 1982; Blumenthal et al. 1984). In the case of hot dark matter, large scale perturbations collapse first, resulting in the formation of structures larger than clusters, followed by subsequent fragmentation into galaxies and clusters (Zeldovich 1970; Doroshkevich et al. 1978). Hybrids of these two scenarios are possible if different types of perturbation are present (Dekel 1983).

The properties which were studied by West and collaborators in the context of these different formation scenarios are: the density profiles of clusters, the occurrence and nature of substructure and the mass radius relation of clusters and, orientation of clusters with respect to larger structures. We briefly summarize the results below.

West et al. (1987) have studied the radial profiles of clusters. The density profiles of the simulated clusters (for $\Omega=1$ ) were found to be similar irrespective of the initial conditions. The projected profiles have logarithmic slopes of about -1.8 at the half-mass radius. The existence of a universal profile suggests that violent relaxation is efficient at erasing traces of the initial conditions from the cluster profile during the first collapse while secondary infall does not significantly affect it.

The existence and nature of substructure in rich clusters has been the subject of much recent debate. West et al. (1988a) have found, using several statistical tests, that the amount of substructure within the main bodies of rich clusters is not very sensitive to initial conditions. In general, the subclustering found in the simulated clusters is much weaker than has sometimes been claimed and in many cases no larger than that expected from random fluctuations. The simulations thus predict that most rich clusters are in a relaxed stage at present. This conclusion depends sensitively on (a) how one determines the present epoch in the simulations and (b) the definition of a "cluster."

West et al. (1988b) have found a well defined radiusmass relation for the simulated clusters, the slope of which 
depends on the form of the initial density fluctuation spectrum. Observationally, they find for a sample of 29 clusters that $R \propto L^{0.51 \pm 0.07}$. If $\mathscr{M} / L$ is roughly the same among clusters and if the distribution of light and mass are similar, then best agreement with the observations is found for an initial spectrum with an effective slope $-1 \leqslant n \leqslant 0$ on the scale of protoclusters as is expected in the cold dark matter scenario.

In general, initial perturbations are expected to be somewhat aspherical (Doroshkevich 1970, Bardeen et al. 1986) and this asphericity will be amplified due to gravitational collapse (Lin et al. 1965). Dekel et al. (1984) have shown that cluster-cluster alignments arise only if sufficient large scale power is present in the initial fluctuation spectrum. Alignments are not produced as a result of tidal interactions between neighboring protoclusters. The result is confirmed by Barnes \& Efstathiou (1987). Alignments of clusters in the context of the cold dark matter (CDM) theory of galaxy formation have been discussed by Bond (1987), Bond \& Szalay (1991) and West et al. (1991). By identifying rich clusters with high peaks in the initial Gaussian density field Bond (1987) and Bond \& Szalay (1991) argue that significant alignments will occur in the CDM model over scales up to $40 \mathrm{Mpc}$. This result is based on analytical methods. West et al. (1991) find, using high resolution $N$-body simulations, that there is a clear tendency for the major axes of neighboring clusters to be aligned with one another over scales up to $60 \mathrm{Mpc}$, in the standard biased cold dark matter model. When the sample is restricted to only those clusters residing in well-defined superclusters significant alignments are found out 120 Mpc.

From the discussion presented above, it is clear that the alignment test is the most sensitive test to use if one is to use clusters of galaxies to test fluctuation spectra. Elongation or ellipticity is a property that survives violent relaxation and thus provides a key link to initial conditions.

\subsection{Previous Observations of Alignments}

We now briefly review the observational data on alignments. It is now well established that position angles of first-ranked cluster galaxies show a strong tendency to be aligned with the major axis of their parent cluster (Sastry 1968; Dressler 1978; Carter \& Metcalfe 1981; Binggeli 1982; Struble 1987). On larger scales, Binggeli (1982) found that the major axes of clusters themselves exhibit a statistical tendency to be aligned with the lines connecting them with neighboring clusters on scales up to $30 \mathrm{Mpc}$ $\left(H_{0}=50 \mathrm{~km} / \mathrm{s} / \mathrm{Mpc}\right)$. This effect has been confirmed by Flin (1987), Rhee \& Katgert (1987), West (1989a,b), Lambas et al. (1990), and Lambas \& West (1991). On the other hand, three studies have found the evidence for alignments to be weak or absent (Struble \& Peebles 1985; Fong et al. 1990; McMillan et al. 1989). The study of McMillan et al. is based on X-ray data.

In related studies, Argyres et al. (1986), Lambas et al. (1988), and Muriel \& Lambas (1989) demonstrated that Shane-Wirtanen galaxy counts in the regions surrounding
Abell clusters tend to be systematically higher along the direction defined by the cluster major axes (or by the position angle of the first-ranked galaxy). These various observations indicate the alignment of structures on scales of several tens of kiloparseconds up to several tens of megaparseconds. West (1989b) has generated a catalog of superclusters using a percolation technique and all clusters with $z \leqslant 0.1$. He has examined the orientations of clusters within superclusters and has shown that if one uses only clusters with reliably determined position angles, clusters exhibit a strong tendency to be aligned with one another on scales of $\sim 60 \mathrm{Mpc}$.

\section{THE DATA}

The goal of this project is thus to study the orientation of clusters of galaxies using a well-defined sample and objective methods for determining position angles. We used three different sets of data for estimating cluster position angles. The first method involves using the galaxy distribution in each cluster to determine the cluster position angle (Rhee et al. 1991). Galaxy position angles can be determined with greater accuracy than cluster position angles (typical uncertainties are $4^{\circ}$ as opposed to $\sim 30^{\circ}-40^{\circ}$ for clusters). There exists a good correlation between the position angle of the first-ranked galaxy and the orientation of its parent cluster. In view of the above two facts, we have studied cluster alignments using the first-ranked galaxy position angles taken from van Kampen \& Rhee (1990). The third method relies on the fact that the gas giving rise to the $\mathrm{x}$-ray emission observed from clusters is in hydrostatic equilibrium and is hence a good tracer of the cluster potential. We have used the $\mathrm{x}$-ray position angles taken from Rhee \& Latour (1991).

The methods and datasets have been described in detail in the papers mentioned above and in Rhee (1989). We work with three methods of measuring position angles and for each dataset have selected the method best suited to producing accurate results. For completeness, we summarize the key points concerning the sample and methods adopted below.

\subsection{The Sample: Optical Data}

The sample consists of 107 Abell clusters having richness class $>0$, redshift $\leqslant 0.1$, right ascension and declination in the range $10^{\mathrm{h}} \leqslant \alpha \leqslant 18^{\mathrm{h}}, \delta>-25^{\circ}$ and $b \geqslant 30^{\circ}$. These constraints essentially define a cone centered on the north galactic pole. Redshifts have been determined for all the clusters in the sample having tenth ranked magnitude $m_{10} \leqslant 16.9$. To determine cluster position angles, we decided to work with galaxy lists obtained from digitized images of the Palomar Sky Survey (PSS). To digitize the red PSS plates, we used the Leiden computer controlled microdensitometer. Areas large enough to include a radius of $2 \mathrm{Mpc}$ surrounding each cluster were digitized. In Table 1 we list the cluster position angles derived from the data. Three positions angles are listed corresponding to three different methods of measuring a position angle. The three methods are (1) the tensor method, (2) the moment 
TABLE 1. The sample

\begin{tabular}{|c|c|c|c|c|c|c|c|c|c|c|c|c|c|c|c|c|c|}
\hline \multirow{2}{*}{$\begin{array}{c}\text { Abell } \\
915\end{array}$} & \multirow[b]{2}{*}{0.0470} & \multicolumn{2}{|c|}{$\mathrm{DR} m_{10}$} & $\begin{array}{l}\mathrm{RS} \\
\text { type }\end{array}$ & $\begin{array}{c}\text { f.r. } \\
\text { gal }\end{array}$ & $\begin{array}{l}\text { BM } \\
\text { class }\end{array}$ & \multicolumn{2}{|c|}{$\begin{array}{l}\text { cluster } \\
\phi_{t} \quad \epsilon_{t}\end{array}$} & $\begin{array}{r}\text { optical } \\
\phi_{m} \epsilon_{m}\end{array}$ & \multicolumn{2}{|c|}{$\phi_{f} \frac{N_{2}}{\sigma_{N_{1}}}$} & \multicolumn{4}{|c|}{ cluster xray data } & \multicolumn{2}{|c|}{$\begin{array}{cc}\text { f.r. } & \text { gal } \\
\phi & \sigma_{\phi}\end{array}$} \\
\hline & & 5 & 117.2 & C & So & II & 19 & 0.32 & 140.18 & 10 & 2.1 & & & & & 2 & 0 \\
\hline 924 & 0.0989 & 5 & $\begin{array}{ll}1 & 17.2\end{array}$ & C & b & II & 80 & 0.11 & 700.16 & 51 & 1.3 & & & & & 99 & 2 \\
\hline 957 & 0.0440 & 4 & $\begin{array}{ll}1 & 15.9\end{array}$ & & E/SO & II & 88 & 0.21 & 1000.11 & 80 & 2.0 & 60 & 12 & 85 & 36 & 89 & 2 \\
\hline 978 & 0.0527 & 3 & 115.6 & $\mathbf{F}$ & cD & I & 135 & 0.22 & 1420.13 & 150 & 3.0 & & & & & 174 & 1 \\
\hline 991 & 0.0880 & 5 & 117.2 & I & b & II & 64 & 0.26 & 840.16 & 60 & 1.5 & & & & & 68 & 1 \\
\hline 994 & 0.0390 & 6 & $\begin{array}{ll}1 & 17.5\end{array}$ & C & & & 138 & 0.15 & 1500.13 & 140 & 1.3 & & & & & 160 & 0 \\
\hline 1020 & 0.0650 & 4 & 116.0 & I & E/S0 & II-III & 153 & 0.21 & $\begin{array}{lll}151 & 0.11\end{array}$ & 160 & 1.5 & & & & & 137 & 5 \\
\hline 1126 & 0.0852 & 4 & 116.0 & B & b & I-II & 110 & 0.22 & 1560.09 & 82 & 2.6 & & & & & 83 & 2 \\
\hline 1169 & 0.0582 & 5 & 116.6 & $\mathbf{F}$ & S/S0 & II & 75 & 0.12 & 480.06 & 45 & 1.8 & & & & & 20 & 5 \\
\hline 1185 & 0.0304 & 2 & 114.3 & C & b & II & 101 & 0.20 & 1110.09 & 100 & 2.5 & 99 & 7 & 76 & 13 & & \\
\hline 1186 & 0.0791 & 5 & 216.5 & C & & II-III & 101 & 0.02 & 280.11 & 100 & 1.8 & & & & & 118 & 9 \\
\hline 1187 & 0.0791 & 3 & 115.6 & I & b & III & 67 & 0.26 & 340.11 & 50 & 1.8 & & & & & 108 & 1 \\
\hline 1190 & 0.0794 & 5 & 216.6 & & S/S0 & I & 171 & 0.26 & 1710.26 & 165 & 2.7 & & & & & 178 & 2 \\
\hline 1213 & 0.0468 & 2 & 114.5 & C & SBc & III & 31 & 0.08 & $\begin{array}{lll}50 & 0.08\end{array}$ & 80 & 0.5 & & & & & 133 & 3 \\
\hline 1216 & 0.0524 & 4 & 116.0 & $\mathbf{F}$ & $\mathbf{E}$ & III & 88 & 0.13 & 1000.07 & 100 & 2.0 & & & & & 133 & 2 \\
\hline 1228 & 0.0350 & 1 & 113.8 & $\mathbf{F}$ & So & II-III & 149 & 0.17 & 1500.16 & 115 & 1.8 & & & & & 55 & 1 \\
\hline 1238 & 0.0716 & 4 & 116.0 & C & b & III & 109 & 0.07 & 100.04 & 10 & 1.0 & & & & & 104 & 3 \\
\hline 1254 & 0.0628 & 3 & 115.3 & & S0/a & III & 73 & 0.09 & 810.09 & 90 & 1.0 & & & & & 180 & 1 \\
\hline 1291 & 0.0530 & 3 & 115.4 & $\mathrm{~F}$ & SBc & III & 177 & 0.16 & 1700.05 & 165 & 1.4 & 62 & 10 & 40 & 26 & & \\
\hline 1317 & 0.0700 & 5 & 216.5 & B & b & I-II & 107 & 0.06 & 1490.09 & 100 & 0.5 & & & & & & \\
\hline 1318 & 0.0566 & 3 & 115.0 & C & Sc & II & 158 & 0.18 & 1660.12 & 170 & 3.0 & & & & & & \\
\hline 1337 & 0.0826 & 5 & 117.2 & L & Ep & II & 67 & 0.23 & 3500.10 & 20 & 2.0 & & & & & 7 & 3 \\
\hline 1344 & 0.0770 & 5 & 116.6 & C & S0/a & II-III & 40 & 0.11 & 490.13 & 80 & 1.0 & & & & & 80 & 1 \\
\hline 1346 & 0.0970 & 5 & 116.8 & & E/SO & II-III & 5 & 0.31 & $1 \quad 80.17$ & 10 & 2.5 & & & & & 6 & 9 \\
\hline 1356 & 0.0698 & 5 & 117.2 & C & $\mathrm{E} / \mathrm{S} 0$ & II-III & 21 & 0.14 & 10.19 & 20 & 2.0 & & & & & 146 & 1 \\
\hline 1365 & 0.0763 & 4 & 115.7 & $\mathbf{F}$ & So & III & 156 & 0.28 & $\begin{array}{lll}371 & 0.11\end{array}$ & 150 & 2.0 & & & & & 14 & 2 \\
\hline 1367 & 0.0215 & 1 & 213.5 & $\mathbf{F}$ & So & II-III & & & & & & 147 & & 149 & 17 & & \\
\hline 1377 & 0.0514 & 3 & 115.0 & & E/S0 & III & $6 i$ & 0.23 & $3 \quad 50 \quad 0.10$ & 70 & 2.0 & 96 & 4 & 95 & 7 & & \\
\hline 1383 & 0.0603 & 4 & 115.7 & I & $\mathrm{S}$ & III & 21 & 0.12 & 280.06 & 140 & 1.0 & & & & & 154 & 2 \\
\hline 1385 & 0.0831 & 5 & 117.2 & C & b & II & 155 & 0.32 & 1480.35 & 150 & 4.0 & & & & & 49 & 3 \\
\hline 1399 & 0.0913 & 4 & 216.0 & & E/SO & III & 161 & 0.08 & 31410.12 & 170 & 1.7 & & & & & 172 & 18 \\
\hline 1412 & 0.0839 & 4 & 215.9 & & E/S0 & III & 112 & 0.16 & 1170.15 & 100 & 2.5 & & & & & 45 & 1 \\
\hline 1424 & 0.0770 & 5 & 116.6 & $\mathrm{C}$ & So & II & 73 & 0.18 & 3710.14 & 65 & 2.0 & & & & & 71 & 1 \\
\hline 1436 & 0.0646 & 3 & 115.4 & I & Sab & III & 31 & 0.42 & 340.21 & 30 & 3.0 & & & & & 92 & 2 \\
\hline 1468 & 0.0844 & 4 & 116.0 & $\mathrm{C}$ & $\mathrm{E}$ & I & 11 & 0.23 & $3 \quad 60.17$ & 10 & 2.5 & & & & & 142 & 8 \\
\hline 1474 & 0.0791 & 4 & 116.0 & & So/E & III & 128 & 0.12 & 1020.03 & 30 & 2.0 & & & & & & \\
\hline 1496 & 0.0941 & 4 & 116.0 & I & So & III & 83 & 0.48 & $3 \quad 910.35$ & 70 & 2.5 & & & & & 14 & 1 \\
\hline 1521 & 0.0940 & 5 & $\begin{array}{ll}116.8 \\
\end{array}$ & $\mathrm{cD}$ & cDsp & II & 121 & 0.15 & 1170.09 & 105 & 1.8 & & & & & 121 & 2 \\
\hline 1526 & 0.0800 & 5 & 116.6 & I & so & II & 125 & 0.22 & 1430.24 & 110 & 1.6 & & & & & 46 & 1 \\
\hline 1539 & 0.0586 & 5 & 217.2 & L & SB0 & II & 25 & 0.11 & 110.10 & 20 & 1.0 & & & & & 149 & 2 \\
\hline 1541 & 0.0892 & 4 & 116.0 & B & b & I-II & 136 & 0.39 & 1440.24 & 140 & 4.0 & & & & & 107 & 1 \\
\hline 1552 & 0.0837 & 5 & 116.6 & B & b & I & 80 & 0.15 & 900.17 & 80 & 1.0 & & & & & 84 & 1 \\
\hline 1609 & 0.0891 & 5 & $\begin{array}{ll}116.8 \\
\end{array}$ & & $\mathrm{E} / \mathrm{S} 0$ & II-III & 80 & 0.26 & $\quad 630.22$ & 75 & 1.5 & & & & & 39 & 1 \\
\hline 1630 & 0.0649 & 5 & $\begin{array}{ll}1 & 16.7\end{array}$ & $\mathrm{C}$ & S/S0 & II-III & 96 & 0.22 & 950.11 & 95 & 2.2 & & & & & 84 & 2 \\
\hline 1644 & 0.0493 & 4 & 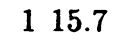 & $\mathrm{cD}$ & $\mathrm{cD}$ & II & 6 & 0.19 & 70.09 & 180 & 2.5 & & & & & 39 & 1 \\
\hline 1650 & 0.0845 & 5 & 217.0 & $\mathrm{cD}$ & cDsp & I-II & 92 & 0.24 & 1140.16 & 90 & 2.0 & 174 & 5 & 11 & 4 & 78 & 1 \\
\hline 1651 & 0.0825 & 4 & 116.0 & $\mathrm{cD}$ & $\mathrm{cD}$ & I-II & 97 & 0.31 & 950.11 & 95 & 3.8 & & & & & 78 & 2 \\
\hline 1656 & 0.0230 & 1 & 213.5 & B & $\mathrm{gE}$ & II & 62 & 0.16 & ; 550.08 & 70 & 4.0 & & & & & & \\
\hline 1668 & 0.0648 & 5 & 116.6 & $\mathrm{cD}$ & $\mathrm{cD}$ & I & 19 & 0.16 & ; 180.16 & & & & & & & 69 & 1 \\
\hline 1691 & 0.0722 & 3 & 115.4 & $\mathrm{cD}$ & $\mathrm{cD}$ & II & 59 & 0.11 & 510.08 & 30 & 1.0 & & & & & 160 & 3 \\
\hline 1749 & 0.0590 & 4 & 116.0 & $\mathrm{cD}$ & $\mathrm{cD}$ & II & 131 & 0.20 & 1130.10 & 130 & 2.5 & & & & & 99 & 1 \\
\hline 1767 & 0.0701 & 4 & 115.7 & $\mathrm{cD}$ & $\mathrm{cD}$ & II & 179 & 0.12 & 1500.07 & 180 & 1.5 & 125 & .43 & 153 & 21 & 144 & 1 \\
\hline
\end{tabular}


TABLE 1. (continued)

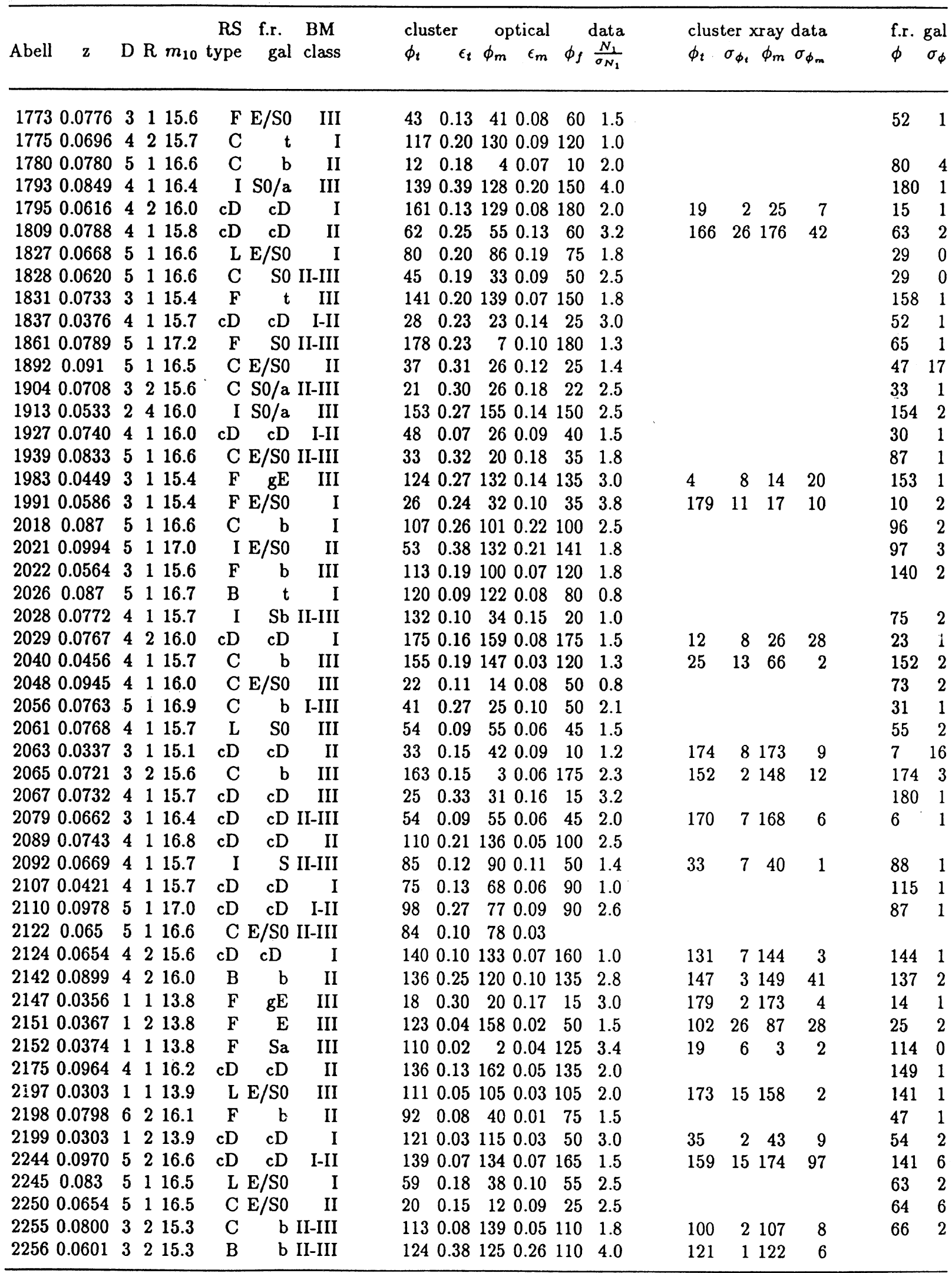


method, (3) Fourier transform of the azimuthal galaxy distribution. These methods have been described in Rhee et al. (1991) and Rhee \& Katgert (1987). The position angle (and associated uncertainty) of the first-ranked galaxy is also listed in Table 1. This position angle was determined using the moment method on the digitized image of each galaxy. For the full details the reader is referred to van Kampen \& Rhee (1990).

We have compared the position angles for the 11 firstranked galaxies in our sample which were also studied by Tucker \& Peterson (1988). The mean difference between our and their quoted position angles is $-0.6^{\circ}$ and the rms difference is $25^{\circ}$. For two galaxies we find a position angle difference $>35^{\circ}$. It is possible that we did not identify the same galaxy as Tucker \& Peterson in these two cases. For the remaining 9 galaxies in our joint sample the mean position angle difference is $-4^{\circ}$ and the rms difference is $13^{\circ}$.

The following abbreviations should be noted in the morphological types of the first-ranked galaxies listed in Table 1:

b-denotes a close binary with an envelope;

$\mathrm{s}$-following only the cD type, denotes a satellite;

$\mathrm{t}$-denotes a triple galaxy with three distinct nuclei;

$\mathrm{g}$-preceding an E or SO designation indicates a "giant" galaxy, in the sense of being larger and brighter compared with other members.

Struble \& Rood (1991) have noted that the redshift for Abell 994 may be that of a foreground galaxy since this is a distance class 6 cluster.

\subsection{The Sample: X-ray Data}

Cluster position angles derived from x-ray data are of great interest for the study of alignments. The Einstein Observatory (HEAO-2) catalog of observations was searched and all clusters in the optical sample which had been observed with the Imaging Proportional Counter (IPC) were selected. This work has been described fully by Rhee \& Latour (1990). We found that 39 of the clusters scanned by us were also observed by Einstein. On closer scrutiny we found that 27 clusters had sufficient signal to noise for the purpose of determining centra, radial profiles, and orientations. The remaining 12 clusters were not used. Cluster position angles were derived from the x-ray data using the moment method. The results of both the tensor and moment methods are listed in Table 1.

\section{ALIGNMENTS}

Binggeli's (1982) original test consisted of comparing the cluster position angle on the sky with the direction to its nearest neighbor defined by the great circle on the sky connecting the two cluster centers. We thus produce a distribution of position angle differences $\delta \theta$. We have also applied a refinement of Binggeli's test suggested by West (1989b), where one calculates $\delta \theta$ for a given cluster to each of its neighbors in a given supercluster. On scales beyond those of clusters, larger complexes (known as su- perclusters) may be identified and it is interesting to study cluster alignments within superclusters.

By applying a percolation algorithm to the $r \geqslant 0$ clusters in the catalog of Abell clusters with known redshift compiled by Struble \& Rood (1987), West (1989b) has generated a catalog of cluster complexes or superclusters, corresponding to a mean overdensity $n / \bar{n} \simeq 11.48$ clusters containing two or more members are identified. West finds that the observed superclusters consist of a mixed population of oblate and prolate objects having axial ratios of order 3:1:1 and 3:3:1. The clusters exhibit a strong tendency to be aligned with other clusters in the same supercluster.

We have repeated the above analysis for the cluster sample presented in this paper. A supercluster catalog was generated in the following manner. Each cluster's right ascension, declination, and redshift was transferred to a Cartesian coordinate system $x=d \cos \delta \cos \alpha, y=d \cos \delta$ $\sin \alpha, z=d \sin \delta$, where $d=c z(1+z / 2) /\left[H_{0}(1+z)^{2}\right]$. The equation used for distance computation is for a deceleration parameter of 0 . Superclusters were identified using a percolation algorithm whereby clusters separated by less than some ciritical distance were linked together. Superclusters are composed of those clusters joined together either directly or indirectly. We have chosen of value of the percolation length of $50 \mathrm{Mpc}$. This is comparable to the observed correlation length of Abell clusters (Bahcall \& Soneira 1983; Klypin \& Kopylov 1983; Postman et al. 1988). We work with the sample listed in Table 1. Seventeen superclusters are generated. There are on average 4 clusters per supercluster (not all the clusters in our sample lie in a supercluster). $63 \%$ of the cluster sample lie in a supercluster. Two superclusters contain more than 10 clusters. Following the method outlined in Rhee et al. (1991), we have calculated cluster position angles and associated uncertainties based on Fourier analysis of the azimuthal distribution of galaxies.

Three tests were made on the $\delta \theta$ distributions. First, we made a test of the hypothesis that the mean $|\delta \theta|$ equals $45^{\circ}$, the mean expected for a uniform distribution on the interval $\left[0^{\circ}, 90^{\circ}\right]$. This was done by evaluating

$$
t=\left(45^{\circ}-\langle|\delta \theta|\rangle\right) \sqrt{N} / \sigma^{\prime}
$$

where $\sigma^{\prime}=\sqrt{\sigma_{\delta \theta}^{2}+\sigma_{\theta_{\mathrm{c}}}^{2}}, \sigma_{\delta \theta}$ is the rms dispersion in $\delta \theta, N$ is the number of cluster pairs and $\sigma_{\theta_{c}}$ is the typical measurement uncertainty in $\theta_{\mathrm{c}}$, the cluster position angle. $\boldsymbol{P}_{t}$, the probability that a value $\geqslant t$ produced by a random distribution is listed in column 6 of Tables 2 and 3 for the various subsamples. Second, the distribution was binned in 9 bins of width $10^{\circ}$ and the $\chi^{2}$ test applied to a uniform distribution. Third, the binomial test was applied to calculate the probability of choosing $m$ clusters out of $n$ clusters with $|\delta \theta|<45^{\circ}$. The results of these tests are shown in Tables 2 and 3.

Three different position angles were used for this study. First, we used cluster position angles derived from the galaxy distribution. Since the cluster first-ranked galaxy alignment is stronger for more elongated clusters and the first- 
TABLE 2. Cluster alignments in superclusters.

\begin{tabular}{|c|c|c|c|c|c|c|}
\hline$d_{\max }$ & $<|\delta \theta|>$ & $\chi^{2} / \nu$ & $P\left(\chi^{2} / \nu\right)$ & $P_{b i n}$ & $P_{t}$ & $N_{\text {pair }}$ \\
\hline \multicolumn{7}{|c|}{ All clusters } \\
\hline $\begin{array}{r}600 \\
60 \\
30 \\
15\end{array}$ & $\begin{array}{l}43.2 \pm 0.5 \\
43.5 \pm 0.9 \\
38.7 \pm 2.1 \\
37.1 \pm 4.1\end{array}$ & $\begin{array}{l}1.91 \\
1.73 \\
1.60\end{array}$ & $\begin{array}{l}0.05 \\
0.05 \\
0.05\end{array}$ & $\begin{array}{l}0.011 \\
0.032 \\
0.020 \\
0.156\end{array}$ & $\begin{array}{l}0.24 \\
0.35 \\
0.14 \\
0.31\end{array}$ & $\begin{array}{r}335 \\
127 \\
45 \\
15\end{array}$ \\
\hline \multicolumn{7}{|c|}{ Clusters with p.a. error $<15^{\circ}$} \\
\hline $\begin{array}{r}600 \\
60 \\
30 \\
15\end{array}$ & $\begin{array}{l}41.9 \pm 1.0 \\
42.6 \pm 1.4 \\
41.0 \pm 2.4 \\
39.8 \pm 5.4\end{array}$ & $\begin{array}{l}2.16 \\
1.42 \\
1.06\end{array}$ & $\begin{array}{l}0.03 \\
0.18 \\
0.39\end{array}$ & $\begin{array}{l}0.008 \\
0.085 \\
0.137 \\
0.311\end{array}$ & $\begin{array}{l}0.01 \\
0.09 \\
0.17 \\
0.25\end{array}$ & $\begin{array}{r}134 \\
54 \\
25 \\
6\end{array}$ \\
\hline \multicolumn{7}{|c|}{ First ranked galaxy } \\
\hline $\begin{array}{r}600 \\
60 \\
30 \\
15\end{array}$ & $\begin{array}{l}39.7 \pm 1.4 \\
39.0 \pm 2.6 \\
33.1 \pm 5.7 \\
25.8 \pm 9.8\end{array}$ & $\begin{array}{l}2.37 \\
0.68 \\
1.50\end{array}$ & $\begin{array}{l}0.01 \\
0.71 \\
0.15\end{array}$ & $\begin{array}{l}0.002 \\
0.027 \\
0.033 \\
0.018\end{array}$ & $\begin{array}{l}<0.01 \\
<0.01 \\
<0.01 \\
<0.01\end{array}$ & $\begin{array}{r}154 \\
50 \\
18 \\
9\end{array}$ \\
\hline \multicolumn{7}{|c|}{ All clusters X-ray data } \\
\hline $\begin{array}{r}600 \\
60 \\
30 \\
15\end{array}$ & $\begin{array}{l}38.8 \pm 0.6 \\
45.6 \pm 0.1 \\
43.3 \pm 0.6 \\
41.3 \pm 1.5\end{array}$ & $\begin{array}{l}1.76 \\
1.89\end{array}$ & $\begin{array}{l}0.08 \\
0.06\end{array}$ & $\begin{array}{l}0.004 \\
0.104 \\
0.182 \\
0.259\end{array}$ & $\begin{array}{r}<0.01 \\
0.36 \\
0.45 \\
0.25\end{array}$ & $\begin{array}{r}142 \\
57 \\
20 \\
10\end{array}$ \\
\hline \multicolumn{7}{|c|}{ Clusters with p.a. error $<15^{\circ} \mathrm{X}$-ray data } \\
\hline $\begin{array}{r}600 \\
60 \\
30 \\
15\end{array}$ & $\begin{array}{l}37.2 \pm 0.9 \\
42.7 \pm 0.5 \\
37.2 \pm 2.6 \\
33.6 \pm 5.6\end{array}$ & $\begin{array}{l}2.56 \\
1.82\end{array}$ & $\begin{array}{l}0.01 \\
0.07\end{array}$ & $\begin{array}{l}0.002 \\
0.102 \\
0.127 \\
0.319\end{array}$ & $\begin{array}{r}<0.01 \\
0.05 \\
<0.01 \\
0.02\end{array}$ & $\begin{array}{r}101 \\
42 \\
12 \\
5\end{array}$ \\
\hline
\end{tabular}

ranked galaxy position angle can be measured more accurately than the cluster position angle, we have used as a second source of position angle the first-ranked galaxy position angles (clusters with spiral first-ranked galaxies were excluded). Third, we have used cluster position angles derived from the $\mathrm{x}$-ray distribution

The data were divided into several subsets to see if any effect present was dependent on pair separation. Using the errors associated with the cluster position angles, we applied the test to the subset of clusters whose position angle could be determined to better than $15^{\circ}$. The alignment effect was also studied as a function of separation between cluster pairs. The data were divided into four subsets of pairs with separation $\leqslant 600,60,30$, and $15 \mathrm{Mpc}$ respectively. Cluster pairs within superclusters were used for the analysis and the results are shown in Table 2. A similar analysis was made by simply calculating the distance to the

TABLE 3. Cluster alignments: nearest neighbor.

\begin{tabular}{|c|c|c|c|c|c|c|}
\hline$d_{\max }$ & $<|\delta \theta|>$ & $\chi^{2} / \nu$ & $P\left(\chi^{2} / \nu\right)$ & $P_{b i n}$ & $P_{t}$ & $N_{\text {pair }}$ \\
\hline \multicolumn{7}{|c|}{ All clusters } \\
\hline $\begin{array}{r}600 \\
60 \\
30 \\
15\end{array}$ & $\begin{array}{l}43.5 \pm 3.6 \\
44.1 \pm 1.2 \\
42.0 \pm 2.1 \\
37.6 \pm 4.5\end{array}$ & $\begin{array}{l}0.95 \\
2.33\end{array}$ & $\begin{array}{l}0.45 \\
0.02\end{array}$ & $\begin{array}{l}0.053 \\
0.069 \\
0.049 \\
0.194\end{array}$ & $\begin{array}{l}0.35 \\
0.39 \\
0.36 \\
0.45\end{array}$ & $\begin{array}{r}101 \\
57 \\
28 \\
12\end{array}$ \\
\hline \multicolumn{7}{|c|}{ Clusters with p.a. error $<15^{\circ}$} \\
\hline $\begin{array}{r}600 \\
60 \\
30 \\
15\end{array}$ & $\begin{array}{l}40.1 \pm 5.3 \\
41.3 \pm 2.5 \\
46.1 \pm 2.0 \\
40.4 \pm 6.4\end{array}$ & & & $\begin{array}{l}0.057 \\
0.097 \\
0.194 \\
0.372\end{array}$ & $\begin{array}{l}0.02 \\
0.11 \\
0.45 \\
0.44\end{array}$ & $\begin{array}{r}24 \\
23 \\
12 \\
4\end{array}$ \\
\hline \multicolumn{7}{|c|}{ First ranked galaxy } \\
\hline $\begin{array}{r}600 \\
60 \\
30 \\
15\end{array}$ & $\begin{array}{l}43.0 \pm 2.3 \\
47.2 \pm 1.4 \\
51.2 \pm 1.2 \\
44.7 \pm 3.6\end{array}$ & $\begin{array}{l}0.95 \\
1.11\end{array}$ & $\begin{array}{l}0.47 \\
0.35\end{array}$ & $\begin{array}{l}0.064 \\
0.117 \\
0.152 \\
0.198\end{array}$ & $\begin{array}{r}<0.01 \\
0.03 \\
<0.01 \\
0.71\end{array}$ & $\begin{array}{l}91 \\
49 \\
24 \\
12\end{array}$ \\
\hline \multicolumn{7}{|c|}{ All clusters X-ray data } \\
\hline $\begin{array}{r}600 \\
60 \\
30 \\
15\end{array}$ & $\begin{array}{l}44.5 \pm 7.7 \\
48.0 \pm 0.4 \\
42.5 \pm 1.0 \\
38.4 \pm 2.9\end{array}$ & & & $\begin{array}{l}0.142 \\
0.127 \\
0.215 \\
0.273\end{array}$ & $\begin{array}{l}0.81 \\
0.15 \\
0.35 \\
0.25\end{array}$ & $\begin{array}{r}26 \\
22 \\
14 \\
7\end{array}$ \\
\hline \multicolumn{7}{|c|}{ Clusters with p.a. error $<15^{\circ} \mathrm{X}$-ray data } \\
\hline $\begin{array}{r}600 \\
60 \\
30 \\
15\end{array}$ & $\begin{array}{l}43.5 \pm 9.0 \\
44.7 \pm 0.4 \\
36.5 \pm 3.0 \\
28.7 \pm 7.8\end{array}$ & & & $\begin{array}{l}0.172 \\
0.175 \\
0.218 \\
0.317\end{array}$ & $\begin{array}{l}0.32 \\
0.85 \\
0.01 \\
0.02\end{array}$ & $\begin{array}{r}18 \\
16 \\
6 \\
3\end{array}$ \\
\hline
\end{tabular}

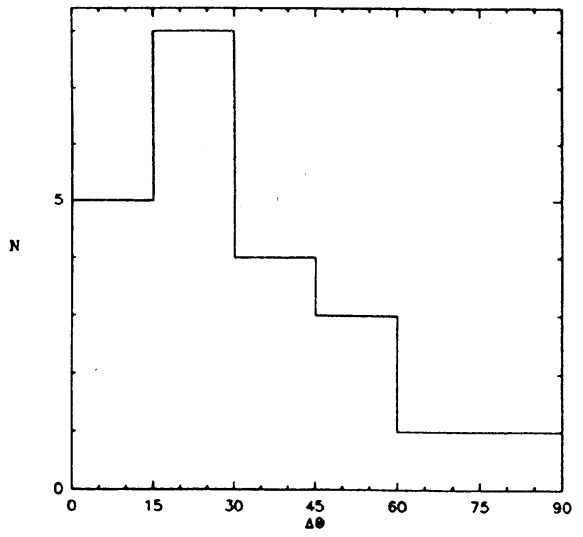

FIG. 1. Histogram of the absolute value of the difference between the position angle of the $\mathrm{CD}$ galaxy and the direction to the nearest neighbor cluster.

cluster nearest neighbor independently as Binggeli (1982) orginally did and the results are shown in Table 3 . The uncertainty listed for $\langle|\delta \theta|\rangle$ in Tables 2 and 3 is the standard error in $\langle|\delta \theta|\rangle$ which is the standard deviation of $\delta \theta$ divided by the square root of the number of pairs.

In Table 3 the results for 20 subsamples are shown. In only 1 out of these 20 cases is $\langle|\delta \theta|\rangle\rangle 45^{\circ}$. Using the $t$-test, the strongest alignment effect is found using the firstranked galaxy position angles. These are also the position angles which are most accurately determined. The position angles derived using $\mathrm{x}$-ray maps also produce significant results if the error in the position angle is less than $15^{\circ}$. It should be noted that both for cluster position angles derived from the $\mathrm{x}$-ray distribution and the galaxy distribution the strength of the effect increases as the position angle uncertainty decreases. Table 2 shows that the three statistical tests produce similar results, namely, that alignments are present for clusters within superclusters at a statistically significant level.

The results shown in Table 3 are not statistically significant, although it is not clear whether this is due to the fact that fewer cluster pairs can be formed for a given $d_{\max }$. It is tempting to speculate that the determining factor is the cluster environment. The results of Table 2 suggest that if a cluster is situated in a high cluster density region alignment effects will be present. Note, however, that we do find an effect to be present for the first-ranked galaxy to the neighboring cluster if this galaxy is a cD galaxy. This effect is shown in a histogram form in Fig. 1 (see also Figs. 2 and 3 ). The figure shows the absolute value of the difference between the $\mathrm{CD}$ position angle and the direction to the nearest neighbor cluster for all $\mathrm{cD}$ galaxies in our sample.

\section{CONCLUSIONS}

The conclusion of the present study is that clusters of galaxies exhibit a clear tendency to point to neighboring clusters if they are members of the same supercluster. In several cases the probability of such an effect arising by chance can be shown to be less than $1 \%$. The effect is 

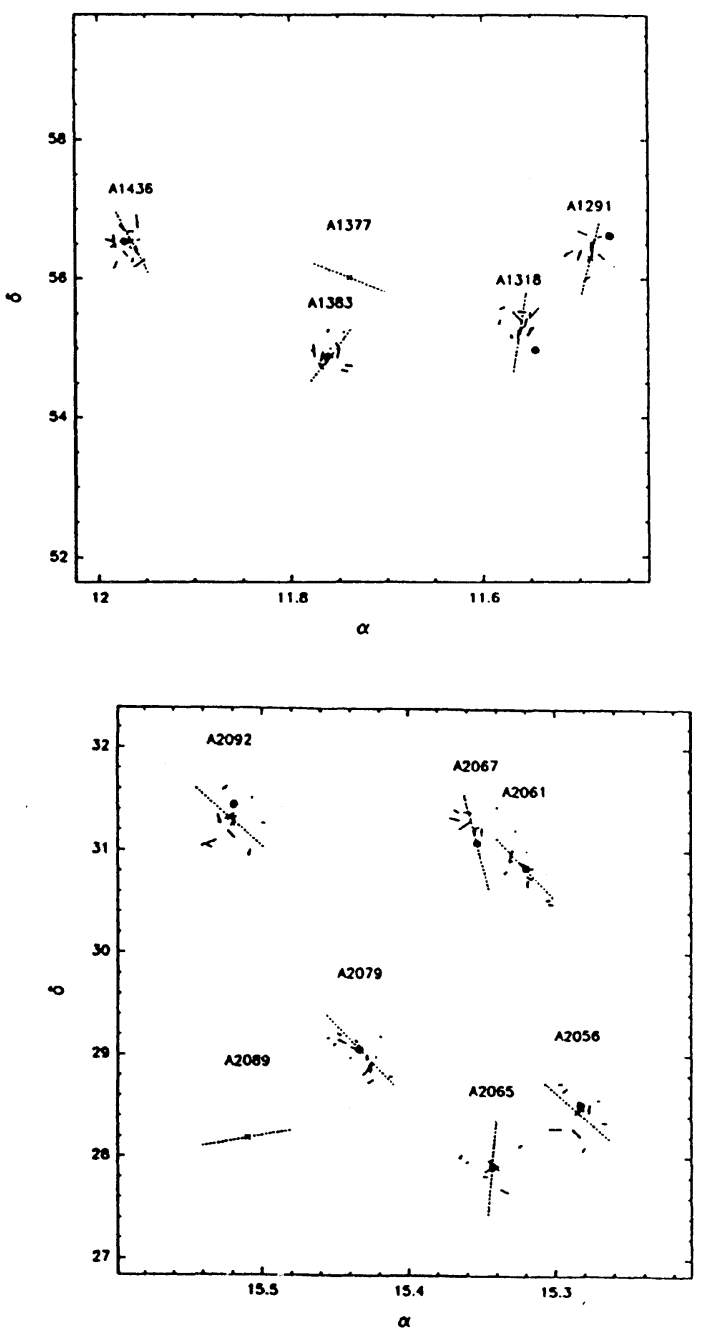

FIG. 2. Maps of two supercluster complexes, displaying the position angles of the member clusters (dashed lines through the cluster centers, the latter marked by a cross) and the position angle distributions of their member galaxies. Galaxy position angles are indicated by line segments with a length proportional to the ellipticity. Shown are (a) the Ursa Major Supercluster, and (b) the Corona Borealis Supercluster.

strongest for the first-ranked galaxy data, weaker for the $\mathrm{x}$-ray data, and weaker for the optical cluster data. In the case of the cluster data, $\langle|\delta \theta|\rangle$ deviates more from $45^{\circ}$ if clusters with position angle errors less than $15^{\circ}$ are used. The same is true of the x-ray data. We find a strong tendency for the first-ranked galaxy to point to the nearest neighbor cluster if the first ranked galaxy is a $\mathrm{cD}$ galaxy.

These results, in conjunction with the well-established alignment of the first-ranked galaxies with their parent cluster, indicate that in high density regions of the universe there exist alignment effects extending from the scale of first-ranked galaxies to that of superclusters or $\sim 3$ decades
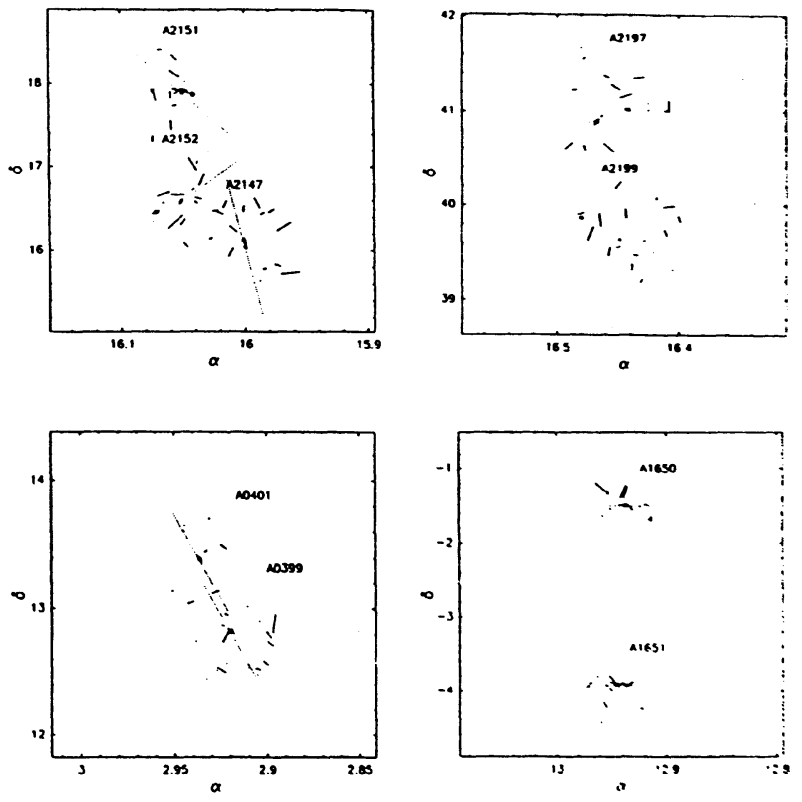

FIG. 3. Maps of (a) the cluster triplet A2147/A2151/A2152, (b) the pair A2197/A2199, (c) A399/A401, and (d) A1650/A1651, displayed as in Fig. 2. The first two are part of the Hercules Supercluster.

in distance. Rhee \& Roos (1990) have made a series of numerical experiments to see if the consistent orientation of matter from the cores of clusters to the scale of superclusters could be reproduced. The collapse of homogeneous ellipsoids was studied to see if the initial large scale elongation is communicated to the inner regions. The results indicate that the central part of the collapsed and virialized system does show the large scale elongation imposed by the initial conditions if the system is prolate. The data of Porter et al. (1991) show that the outer isophotes of brightest elliptical galaxies in Abell clusters are so flattened at large radii that these galaxies cannot be a population of oblate spheroids. In addition, the $N$-body simulations reproduced the increasing ellipticity as a function of radius that is observed for brightest cluster ellipticals.

West et al. (1991) have made a study of alignments using high resolution $N$-body simulations with initial conditions generated following the prescription of the cold dark matter model. They find a clear tendency for the major axes of neighboring clusters to be aligned over scales up to $60 \mathrm{Mpc}$. When the sample is restricted to only those clusters residing in well-defined superclusters, significant alignments are found up to $120 \mathrm{Mpc}$ or more. The results of the present study do not pose a serious challenge for the cold dark matter model, although the model does appear to be inconsistent with the cluster-cluster correlation function and the existence of large scale streaming velocities.

G. R. acknowledges support from the Netherlands Foundation for Astronomical Research (ASTRON) through Grant No. 782-373-036. 


\section{REFERENCES}

Argyres, P. C., Groth E. J., Peebles, P. J. E., \& Struble, M. F. 1986, AJ, 91, 471

Bahcall, N. A., \& Soneira, R. M. 1983, ApJ, 270, 20

Bardeen, J. M., Bond, J. R., Kaiser, N., \& Szalay, A. S. 1986, ApJ, 304, 15

Barnes, J., \& Efstathiou G. 1987, ApJ, 319, 575

Binggeli, B. 1982, A\&A, 107, 338

Blumenthal, G. R., Faber, S. M., Primack, J. R., \& Rees, M. J. 1984, Nature, 311, 517

Bond, J. R. 1987, in Nearly Normal Galaxies, edited by S. Faber (Springer, New York), p. 388

Bond, J. R., \& Szalay, A. S. 1991, in preparation

Carter, D., \& Metcalfe, N. 1981, MNRAS, 191, 325

Dekel, A. 1983, ApJ, 264, 373

Dekel, A., West, M. J., \& Aarseth, S. J. 1984, ApJ, 279, 1

Doroshkevich, A. G. 1970, Afz, 6, 320

Doroshkevich, A. G., Shandarin, S., \& Saar, E. 1978, MNRAS, 184, 64 Dressler, A. 1978, ApJ, 226, 55

Flin, P. 1987, MNRAS, 228, 941

Fong, R., Stevenson, P. R. F., \& Shanks, T. 1990, MNRAS, 242, 146 Klypin, A. A., \& Kopylov, A. J. 1983, SvAL, 14, 7

Lambas, D. G., Groth, E. J., \& Peebles, P. J. E. 1988, AJ, 95, 975

Lambas, D. G., Nicotra, M., Muriel, H., \& Ruiz, L. 1990, AJ, 100, 1006

Lambas, D. G., \& West, M. J. 1991, preprint

Lin, C. C., Mestel, L., \& Shu, F. H. 1965, ApJ, 142, 1431

McMillan, S. L. W., Kowalski, M. P., \& Ulmer, M. P. 1989, ApJS, 70, 723
Muriel, H., \& Lambas, D. G. 1989, AJ, 98, 995

Peebles, P. J. E. 1982, ApJ, 258, 415

Porter, A. C., Schneider, D. P., \& Hoessel, J. G. 1991, AJ, 101, 1561

Postman, M., Geller, M. J., \& Huchra, J. P. 1988, AJ, 95, 367

Rhee, G. F. R. N. 1989, Ph.D. thesis, University of Leiden

Rhee, G. F. R. N., \& Katgert, P. 1987, A\&A, 183, 217

Rhee, G. F. R. N., \& Latour, H. J. 1991, A\&A, 243, 38

Rhee, G. F. R. N., \& Roos, N. 1990, MNRAS, 243, 629

Rhee, G. F. R. N., van Haarlem, and Katgert, P. 1991, A\&AS (in press)

Sastry, G. N. 1968, PASP, 80, 252

Struble, M. F. 1987, ApJ, 317, 668

Struble, M. F., \& Peebles, P. J. E. 1985, AJ, 90, 582

Struble, M. F., \& Rood, H. J. 1987, ApJS, 63, 543

Struble, M. F., \& Rood, H. J. 1988, ApJ, 323, 468

Struble, M. F., \& Rood, H. J. 1991, ApJS, 77, 363

Tucker, G. S., \& Peterson, J. B. 1988, AJ, 95, 298

van Kampen, E., \& Rhee, G. F. R. N. 1990, A\&A, 237, 283

West, M. J. 1989a, ApJ, 344, 535

West, M. J. 1989b, ApJ, 347, 610

West, M. J., Dekel, A., \& Oemler, A. 1987, ApJ, 316, 1

West, M. J., Dekeler, A., \& Oemler, A. 1989, ApJ, 336, 46

West, M. J., Oemler, A., \& Dekel, A. 1988a, ApJ, 327, 1

West, M. J., Oemler, A., \& Dekel, A. 1988b, preprint

West, M. J., Villumsen, J. V., \& Dekel, A. 1991, preprint

Zeldovich, Ya., B. 1970, A\&A, 5, 84 